\title{
Translating neuroimaging findings into psychiatric practice
}

Thomas J. Reilly and Philip K. McGuire

\author{
Summary \\ Although translational medicine has become a priority \\ for medical science, advances in neuroscience \\ have failed to be translated for the benefit of \\ patients. In populations at high risk of psychosis, \\ neuroimaging could stratify those mostly likely to develop
}

psychosis. This is an example of potentially translatable psychiatry.

Declaration of interest

None.
Thomas J. Reilly (pictured) is an academic foundation doctor at University of Glasgow and NHS Greater Glasgow and Clyde. Philip K. McGuire is Head of Psychosis Studies at the Institute of Psychiatry, and Clinical Director of OASIS, an early intervention service for those at risk of psychosis.

Since the landmark discoveries of antidepressants, antipsychotics and mood stabilisers midway through the last century, the progress of clinical psychiatry has faltered. Psychiatric diagnosis is still subjective and symptom-based, because the underlying pathophysiology remains largely unknown. Psychiatric treatment, either pharmacological or psychological, is crude compared with more targeted forms of medical therapy. In contrast, basic neuroscience, especially research on processes and systems directly relevant to psychiatry, has flourished. Today, we know far more about the human brain than 50 years ago, yet the practice of psychiatry has changed little: scientific advances have not been translated into clinical practice.

\section{Translational medicine}

In theory, the goal of basic medical science is to improve the health of patients. In recent years, the effort to quickly shift scientific discoveries 'from the bench to the bedside' has become formalised in translational medicine. This is now a fashionable term; there are new journals, Masters degrees, university departments and governmental initiatives devoted to translational medicine. The thrust of translational research is to gain clinical benefit from discovery, fast. Unfortunately, examples of translational psychiatry are conspicuous by their absence. All the major advances in psychiatric treatment, such as the use of antipsychotic drugs, have been serendipitous rather than hypothesis driven. Nevertheless, there is no good reason why psychiatry cannot exploit advances in neuroscience to inform clinical practice. Neuroimaging is one promising form of basic science which could help translate research findings into benefits for patients with mental illnesses, and is a useful exemplar for translational psychiatry.

\section{Neuroimaging in psychiatry and medicine}

In the mid-1970s, computed tomography was used to identify cerebral infarctions for the first time. ${ }^{1}$ Around this time the same imaging modality demonstrated that the brains of people with chronic schizophrenia are neuroanatomically different from healthy controls. ${ }^{2}$ Today, the management of a patient with suspected stroke would optimally include a battery of imaging investigations such as computed tomography, magnetic resonance imaging, magnetic resonance angiography and transcranial carotid Doppler ultrasound. Despite enormous advances in image acquisition and analysis, neuroimaging still plays almost no role in the clinical management of psychiatric patients. For example, although there are clear group differences between patients with psychiatric disorders and healthy controls, brain scanning does not usually contribute to making a psychiatric diagnosis. The divergence between the clinical application of neuroimaging in psychiatric and neurological disorders is stark. This is in part because brain scans in psychiatric illness are not qualitatively different from normal controls, even though at a group level they are quantitatively. Furthermore, the abnormalities are usually distributed across the brain, rather than limited to a particular focal region. It is thus difficult for a neuroradiologist to recognise a brain scan from a patient with schizophrenia, whereas they would have little difficulty if the patient had been affected by a stroke. Although, by comparing many scans, we can differentiate a group of people with schizophrenia from a group who do not have the disease. The divergence between the use of neuroimaging in psychiatric and medical disorders is also partly due to a failure to translate the science of brain imaging to clinical practice.

\section{Neuroimaging the transition to psychosis}

One of the most promising clinical applications of neuroimaging in psychiatry is in predicting the later development of psychosis. The onset of psychotic disorders such as schizophrenia is preceded by a high-risk phase, a clinical syndrome characterised by psychotic symptoms and a decline in overall function. Up to a third of people with this syndrome will develop a psychotic disorder within 2 years. ${ }^{3}$ However, it is impossible to predict which individuals will go on to develop psychosis on clinical grounds. If it were possible to stratify samples at the high-risk stage, the subgroup that are at most risk of developing psychosis could be selectively given treatments that are designed to reduce this risk. Conversely, unnecessary intervention could be avoided in individuals who are destined to recover.

Recent neuroimaging research has revealed that there are many differences between the subgroup of people at high risk who later develop psychosis and the majority who do not. Although these groups are clinically indistinguishable, the structure, function, chemistry and connectivity of their brains are significantly different. At first presentation, individuals who 
subsequently develop psychosis have less grey matter in the prefrontal, cingulate and temporal cortex ${ }^{4,5}$ altered function in these regions, ${ }^{6}$ and elevated subcortical dopamine synthesis capacity. $^{7}$ As these individuals make the transition to psychosis, there is a longitudinal progression in baseline abnormalities. ${ }^{4,7}$

Neuroimaging has thus shown that alterations in brain structure, function, chemistry and connectivity pre-date the onset of psychosis. However, if these discoveries are to have more than just academic importance, they must be translated for clinical benefit.

\section{Translating neuroimaging science}

A key challenge for translating neuroimaging findings into clinical practice is that research has identified differences between groups of patients but clinical decisions have to be made on the basis of data from an individual. Statistical methods have the potential to overcome this problem. Machine learning is a statistical approach that can be used to estimate the likelihood that a given individual belongs to one clinical group or another; for example, high-risk patients who later develop psychosis, or high-risk patients who will not. ${ }^{8}$ This approach has already been used with some success in predicting the individual risk of transition to psychosis, ${ }^{9}$ and the course of illness after the onset of psychosis. ${ }^{8}$ To date, it has mainly been applied using a single type of imaging data, but its accuracy may be improved by combining data from different imaging modalities, and incorporating neurocognitive and genetic measures in the analysis. ${ }^{10}$

In the future, psychiatrists may thus be able to use quantitatively analysed brain scans and other biomarkers as well as their own clinical judgement to stratify patients in terms of clinically meaningful outcomes. This would allow psychiatrists to tailor the form of treatment to the needs of each individual patient.

\section{Delivering on promise}

Translational psychiatry requires psychiatrists to operate outwith their comfort zone in embracing research developments. We owe it to our patients to deliver on the promises which modern neuroscience offers. Unravelling the intricacies of the human brain is meaningless if we continue to employ 20th-century clinical practice in the 21st century. The inherent complexity and uncertainty of mental illness make this task more difficult than in other fields of medicine, so it requires a concerted effort from individuals, institutions and governments. Time and money need to be invested to translate basic research into clinical practice, but the reward of transforming the lives of those living with psychiatric disorders makes it well worth the expense.

Thomas J. Reilly, BSC, MBChB, Academic Foundation Programme, University of Glasgow, UK; Philip K. McGuire, PhD, FRCPsych, Institute of Psychiatry, King's College London, UK

Correspondence: Thomas J. Reilly, University of Glasgow, University Avenue, Glasgow G12 8QQ, UK. Email: t.reilly@nhs.net

First received 7 Apr 2013, accepted 25 Jun 2013

\section{References}

1 Paxton R, Ambrose J. Proceedings: a review of the results of EMI scanning in the first 650 patients. Br J Radiol 1974; 47: 515

2 Johnstone EC, Crow TJ, Frith CD, Husband J, Kreel L. Cerebral ventricular size and cognitive impairment in chronic schizophrenia. Lancet 1976; 2: 924-6.

3 Fusar-Poli P, Bonoldi I, Yung AR, Borgwardt S, Kempton MJ, Valmaggia L, et al. Predicting psychosis: meta-analysis of transition outcomes in individuals at high clinical risk. Arch Gen Psychiatry 2012; 69: 220-9.

4 Pantelis C, Velakoulis D, McGorry P, Wood SJ, Suckling J, Phillips $\mathrm{U}$, et al. Neuroanatomical abnormalities before and after onset of psychosis: a cross-sectional and longitudinal MRI comparison. Lancet 2003; 361: 281-8.

5 Mechelli A, Reicher A, Meisenzahl E, Tognin S, Wood SJ, Borgwardt SJ, et al. Neuroanatomical abnormalities that predate the onset of psychosis: a multicentre study. Arch Gen Psychiatry 2011; 68: 489-95.

6 Allen P, Luigies J, Howes OD, Egerton A, Hirao K, Valli I, et al. Transition to psychosis associated with prefrontal and subcortical dysfunction in ultra high-risk individuals. Schizophr Bull 2012; 38: 1268-76.

7 Howes O, Bose S, Turkheimer F, Valli I, Egerton A, Stahl D, et al. Progressive increase in striatal dopamine synthesis capacity as patients develop psychosis: a PET study. Mol Psychiatry 2011; 16: 885-6.

8 Mourao-Miranda J, Reinders AA, Rocha-Rego V, Lappin J, Rondina J, Morgan C, et al. Individualized prediction of illness course at the first psychotic episode: a support vector machine MRI study. Psychol Med 2012; 42: 1037-47.

9 Koutsouleris N, Meisenzahl EM, Davatzikos C, Bottlender R, Frodl T, Scheuerecker $\mathrm{J}$, et al. Use of neuroanatomical pattern classification to identify subjects in at-risk mental states of psychosis and predict disease transition. Arch Gen Psychiatry 2009; 66: 700-12.

10 Koike S, Takano Y, Iwashiro N, Satomura Y, Suga M, Nagai, et al. A multimodal approach to investigate biomarkers for psychosis in a clinical setting: The integrative neuroimaging studies in schizophrenia targeting for early intervention and prevention (IN-STEP) project. Schizophr Res 2013; 143: $116-24$ 